\title{
Blood mass spectrometry detects residual disease better than standard techniques in light-chain amyloidosis
}

Angela Dispenzieri ${ }^{1,2}$, Bonnie Arendt ${ }^{2}$, Surendra Dasari ${ }^{2}$, Mindy Kohlhagen ${ }^{2}$, Taxiarchis Kourelis ${ }^{1,2}$, Shaji K. Kumar (1) Nelson Leung $\mathbb{B}^{1,3}$, Eli Muchtar $\mathbb{D}^{1}$, Francis K. Buadi ${ }^{1}$, Rahma Warsame ${ }^{1}$, Robert A. Kyle ${ }^{1,2}$, Martha Q. Lacy ${ }^{1}$, David Dingli' Prashant Kapoor', Wilson I. Gonsalves', Ronald S. Go (1)', Suzanne R. Hayman', Yi Lisa Hwa', Amy Fonder ', Miriam Hobbs', Dragan Jevremovic ${ }^{2}$, John A. Lust ${ }^{1}$, Steven Zeldenrust', Steve J. Russell', S. Vincent Rajkumar (1), Morie A. Gertz and David Murray ${ }^{2}$

\begin{abstract}
In patients with immunoglobulin light-chain (AL) amyloidosis, depth of hematologic response correlates with both organ response and overall survival. Our group has demonstrated that screening with a matrix-assisted laser desorption/ionization-time-of-flight (TOF) mass spectrometry (MS) is a quick, sensitive, and accurate means to diagnose and monitor the serum of patients with plasma cell disorders. Microflow liquid chromatography coupled with electrospray ionization and quadrupole TOF MS adds further sensitivity. We identified 33 patients with AL amyloidosis who achieved amyloid complete hematologic response, who also had negative bone marrow by six-color flow cytometry, and who had paired serum samples to test by MS. These samples were subjected to blood MS. Four patients (12\%) were found to have residual disease by these techniques. The presence of residual disease by MS was associated with a poorer time to progression (at 50 months $75 \%$ versus $13 \%, p=0.003$ ). MS of the blood outperformed serum and urine immunofixation, the serum immunoglobulin free light chain, and six-color flow cytometry of the bone marrow in detecting residual disease. Additional studies that include urine MS and next-generation techniques to detect clonal plasma cells in the bone marrow will further elucidate the full potential of this technique.
\end{abstract}

\section{Introduction}

Immunoglobulin light-chain (AL) amyloidosis is a lifethreatening illness. Depth of hematologic response correlates with both organ response and overall survival. Our group has demonstrated that screening with a matrixassisted laser desorption/ionization-time-of-flight (TOF) mass spectrometry (MASS-FIX) is a quick, inexpensive, and accurate means to diagnose and monitor the serum and urine of patients with plasma cell disorders ${ }^{1-3}$.

Correspondence: Angela Dispenzieri (dispenzieri.angela@mayo.edu) ${ }^{1}$ Department of Internal Medicine, Division of Hematology, Mayo Clinic Rochester, Rochester, MN, USA

${ }^{2}$ Department of Laboratory Medicine and Pathology, Mayo Clinic Rochester, Rochester, MN, USA

Full list of author information is available at the end of the article.
Samples can be reflexed to microflow liquid chromatography coupled with electrospray ionization and quadrupole TOF mass spectrometry (ESI-TOF) ${ }^{4,5}$. Because these techniques provide a mass/charge for a given patient's monoclonal protein, they can provide greater sensitivity and specificity to monitor for residual disease ${ }^{5}$. Our goal was to assess mass spectrometry performance in patients with AL amyloidosis who have been classified as amyloid complete hematologic response using consensus criteria $^{6,7}$ and six-color flow cytometry of bone marrow.

\section{Methods}

The Mayo Foundation Institutional Review Board (IRB) approved the study. All patients gave written informed consent to have their medical records reviewed and 
samples analyzed according to IRB requirements and federal regulations. Patients were eligible for this retrospective study if they: (1) were diagnosed with AL amyloidosis between January 2000 and May 2015; (2) were classified as amyloidosis complete hematologic response by immunofixation electrophoresis (IFE), serum free light chain (FLC) by consensus criteria; ${ }^{6,7}$ (3) had a negative bone marrow by six-color flow cytometry; and (4) had both a stored research sample prior to starting a line of therapy and a repeat sample while in complete hematologic response. The diagnosis of amyloidosis was made by Congo red with green birefringence under polarized light; the typing of the amyloid was with immunohistochemical stains or proteomics ${ }^{8,9}$. Supplementary Figure 1 is a consort diagram illustrating patient selection. Median time from institution of therapy to complete response (CR) sample was 18 months (interquartile range 9.1, 20 months).

The serum IFE (SIFE), urine IFE (UIFE), FLC, and bone marrow measurements were done as part of routine clinical practice as previously described ${ }^{4,5}$. Urine samples were concentrated to a maximum of $200 \times$ to achieve final concentrations of urine protein between 20 and $80 \mathrm{~g} / \mathrm{L}^{4,5}$. The FLC assay (Freelite ${ }^{\mathrm{TM}}$, The Binding Site Ltd.) was performed on a Siemens BNII nephelometer ${ }^{10}$, and an abnormal FLC result was defined as an abnormal FLC $K / \lambda$ ratio. Bone marrow clonality was determined by six-color flow cytometry ${ }^{11}$. This method has sensitivity of $\sim 10^{-4}$ to $10^{-5}$.

For MASS-FIX, immunoglobulins were enriched from serum using camelid-derived nanobodies directed against the heavy-chain constant domains of IgG, IgA, and IgM or the light-chain constant domains of $\mathrm{\kappa}$ and $\lambda$ (Thermo Fisher Scientific) $)^{4,5}$. The +1 and +2 charge states of the light chains and heavy chains were measured by configuring the mass spectrometer to analyze ions between an $m / z$ of $9000-32,000 \mathrm{Da}$.

Samples were subjected to an additional liquid chromatography electrospray ionization tandem mass spectrometric analysis on a Q-TOF mass spectrometer [SCIEX TripleTOF 5600 quadrupole MS (Vaughan, ON, Canada) operating in ESI-positive mode with a Turbo V dual ion source with an automated calibrant delivery system $]^{12}$.

Resulting mass spectra of each sample were visually inspected by three independent reviewers (D.M., B.A., and A.D.), in order to detect and isotype any $M$ proteins present in the patient. All reviewers were blinded to clinical information at the time of the spectral analysis. Clinical histories were reviewed by A.D.

Hematologic progression was defined according to the consensus criteria ${ }^{6,7}$ with the addition of including commencement of a new line of clone-directed therapy based on worsening of hematologic parameters as progression.
Death, in the absence of hematologic progression, was not considered to be a progression. Overall survival and time to progression were calculated according the methods of Kaplan-Meier and differences were determined by Wilcoxon. Statistics were performed using JMP PRO 14.1.0 (SAS, NC).

\section{Results}

Thirty-three patients met the criteria of CR by blood and bone marrow, and their baseline characteristics are shown in Table 1 . Median age was 56 years. Fifty-five percent were male, and all were Caucasian. No test performed perfectly at baseline with the exception of the MASS-FIX due to inclusion requirements. The positive baseline results for the other assays are shown in

\section{Table 1 On-study and treatment characteristics.}

\begin{tabular}{|c|c|}
\hline & All patients $(n=33)$ \\
\hline Age (years), median (range) & $56(44,81)$ \\
\hline Male gender, $n$ (\%) & $18(55)$ \\
\hline Creatinine (mg/dL), median (range) & $1.1(0.7,5.4)$ \\
\hline Creatinine $\geq 2 \mathrm{mg} / \mathrm{dL}, n(\%)$ & $3(9)$ \\
\hline iFLC (mg/dL), median (range) & $13.2(2.0,195)$ \\
\hline Abnormal FLC ratio ${ }^{\mathrm{a}, \mathrm{b}}, n$ (\%) & $27(84)$ \\
\hline SIFE positive, $n(\%)$ & $28(85)$ \\
\hline SIFE HC ${ }^{c}, G / A / D / N e g, n$ & $12 / 9 / 1 / 12$ \\
\hline MALDI positive, $n(\%)$ & $33(100)$ \\
\hline UIFE positive, $n$ (\%) & $23^{d}(79)$ \\
\hline BMPC (\%) (range) & $9(2,40)$ \\
\hline Amyloid type: $k / \lambda, n(\%)$ & $5 / 28(15 / 85)$ \\
\hline Mayo stage $2004^{b}, I$, II, IIIa /IIIb, n & $12 / 11 / 8 / 0$ \\
\hline Mayo stage $2012^{b}, I, I I, I I I, I V, n$ & $13 / 8 / 4 / 6$ \\
\hline Organ involvement, $H, K, L, O, n$ & $10 / 25 / 1 / 4$ \\
\hline \multicolumn{2}{|l|}{ Line of therapy } \\
\hline First line & $31(93)$ \\
\hline Second line & $2(7)$ \\
\hline \multicolumn{2}{|l|}{ Therapies } \\
\hline ASCT, no induction & $19(57)$ \\
\hline ASCT induction & $9(27)$ \\
\hline Mel-Dex & $2(6)$ \\
\hline CVD & $2(6)$ \\
\hline CRD & $1(3)$ \\
\hline
\end{tabular}

$H$ heart, $K$ kidney, $L$ liver, $O$, other which includes $G l$, nerve, and lung. a Outside normal range, which is 0.26 and $1.65 \mathrm{mg} / \mathrm{dL}$.

${ }^{\mathrm{b}}$ One missing data.

'One biclonal patient $(\mathrm{L}+\mathrm{GK})$ counted twice.

${ }^{\mathrm{d}}$ Four missing data. 
Supplementary Fig. 2: SIFE, 85\%; UIFE, 79\%; and abnormal FLC ratio, $84 \%$. Five SIFE-negative patients were positive by MASS-FIX and ESI-TOF, another SIFE negative was found to have a monoclonal $\lambda$ by ESI-TOF and UIFE, and four SIFE negatives had abnormal FLC ratios. Apart from disagreements between positive and negative, isotype discrepancies were seen between SIFE and MASS-FIX in only one instance: IgG $\lambda$ by SIFE, but free $\lambda$ by MASS-FIX; ESI-TOF detected the IgG $\lambda$. No isotype discrepancies were observed between FLC and MASS-FIX apart from the disagreements in four cases in which MASS-FIX was positive and FLC negative. There were eight disagreements in positive-negative calls between FLC and SIFE.

At $\mathrm{CR}$ assessment, by definition all patients had negative SIFE, negative UIFE, normal FLC ratio, and a negative bone marrow by six-color flow cytometry. By MASSFIX and ESI-TOF, respectively, two and four patients were found to have their original monoclonal protein detected at CR determination (Figs. 1 and 2). Another eight had monoclonal proteins that did not coincide with their original protein at CR measurement, consistent with transient post-therapy oligoclonal banding. Therefore, a total 12\% (4 of 33) of patients who were thought to be in CR by high-resolution bone marrow flow cytometry, SIFE, UIFE, and FLC were found to have residual disease by mass spectrometric techniques of the blood.
We next evaluated the effect of a positive result by mass spectrometry on time to progression and overall survival (Fig. 3). Median follow-up for the cohort was 116 months (range 37, 183 months). In the mass spectrometry-positive group, by 50 months $75 \%$ of patients had progression events in contrast to $13 \%$ in the mass spectrometry-negative group, $p=0.003$ (Fig. 4a). Respective 10 -year overall survival rates were $62 \%$ and $83 \%, p=$ not significant (Fig. 4b). Twenty-two patients achieved organ response, seven did not, and four were not accessible due to non-measurable disease (two gastrointestinal, one pulmonary, and one nerve). Of the four positive mass spectrometry patients, there were two who had organ response, one who did not, and another who did not have measurable disease (pulmonary disease only).

\section{Discussion}

We have demonstrated the value of MASS-FIX in detecting residual disease in patients with AL amyloidosis. MS detected residual disease among AL amyloidosis patients in hematologic CR, not only in the context of negative blood and urine IFE and serum FLC but also in the context of a negative bone marrow employing sixcolor flow cytometry, which has approximately one order of magnitude less sensitivity than the next-generation flow cytometry and two orders of magnitude than the

\begin{tabular}{|c|c|c|c|c|c|c|c|c|c|c|c|c|c|c|c|c|c|c|c|c|c|c|c|c|c|c|c|c|c|c|c|c|c|}
\hline \multicolumn{3}{|c|}{$\square$ Negative } & \multicolumn{11}{|c|}{$\square$ Oligoclonal, alternate band } & \multicolumn{14}{|c|}{$\square$ Positive for original clone (not CR) } & \multicolumn{6}{|c|}{$\square$ Not done } \\
\hline Subject number & 1 & 2 & 3 & 4 & 5 & 6 & 7 & 8 & 9 & 10 & 11 & 12 & 13 & 14 & 15 & 16 & 17 & 18 & 19 & 20 & 21 & 22 & 23 & 24 & 25 & 26 & 27 & 28 & 29 & 30 & \begin{tabular}{l|l}
31 & 3 \\
\end{tabular} & \begin{tabular}{l|l}
32 & 3 \\
\end{tabular} & 3 \\
\hline IFE at Baseline & AL & AL & AL & AL & AL & AL & AL & AL & AL & GL & $\mathrm{GL}$ & GL & $\mathrm{GL}$ & GL & GL & GL & L & $\mathrm{L}$ & $\mathrm{L}$ & $\mathrm{L}$ & L & $\mathrm{L}$ & $\begin{array}{l}\mathrm{L}+ \\
\mathrm{GK}\end{array}$ & $\mathrm{DL}$ & $\mathrm{N}^{*}$ & $\mathrm{~N}^{\star}$ & $\mathrm{N}^{\star}$ & $\mathrm{N}^{*}$ & GK & GK & & $\mathrm{GK} / \Lambda$ & \\
\hline FLC clone at CR & & & & & & & & & & & & & & & & & & & & & & & & & & & & & & & & & \\
\hline SIFE at CR & & & & & & & & & & & & & & & & & & & & & & & & & $\mathrm{GK}$ & & & & & & & & \\
\hline MASS-FIX at CR & & & & & GK & & & GK & AL & $L^{n}$ & & & & & & $L^{\wedge}$ & $L^{n}$ & & & & & & & & GK & & & & & GK' & & & \\
\hline ESI-TOF at CR & & $\mathrm{AL}$ & & AL & & & & GK & AL & $L^{n}$ & & & & & & $\mathrm{GL}^{\wedge}$ & $L^{n}$ & & & & & & & & & $\mathrm{~L}^{n}$ & & & & GK' & & & \\
\hline
\end{tabular}

Fig. 1 Grading of complete response using various techniques. Any SIFE with a subsequent isotype discordant with baseline represents oligoclonal banding and unrelated to the original clone. ${ }^{*}$ L by MASS-FIX; ${ }^{*}$ AK by MASS-FIX; $\wedge$ different mass, so considered negative.

\begin{tabular}{|c|c|}
\hline Kappa & Lambda \\
\hline 2,500 & 3,000 \\
\hline $\begin{array}{l}2,000 \\
1,500\end{array}$ & 2,000 \\
\hline $\begin{array}{r}1,000 \\
500\end{array}$ & 1,000 \\
\hline & 0 \\
\hline 1,000 & 1,000 \\
\hline 800 & 800 \\
\hline 600 & 600 \\
\hline 400 & 400 \\
\hline 200 & 200 \\
\hline 0 & \\
\hline
\end{tabular}

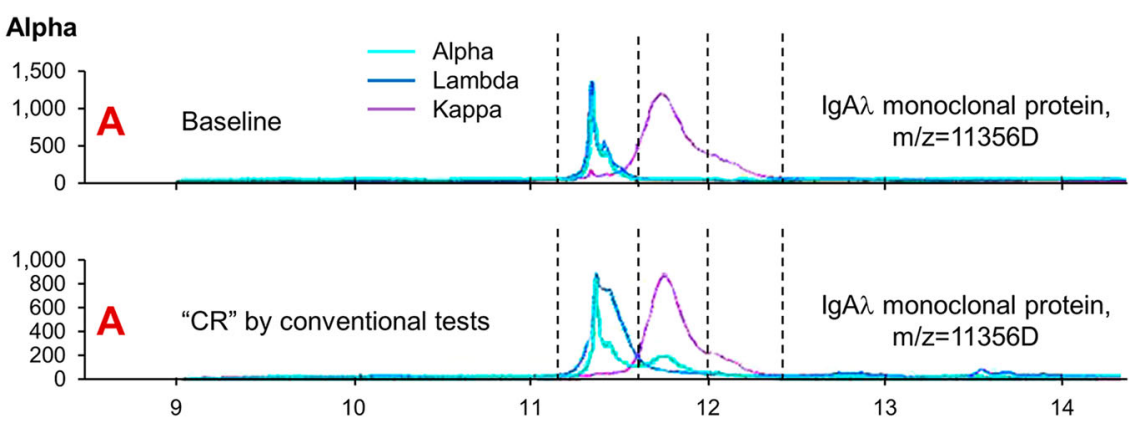

Fig. 2 Example of positive MASS-FIX in patient otherwise deemed to be in complete response. 


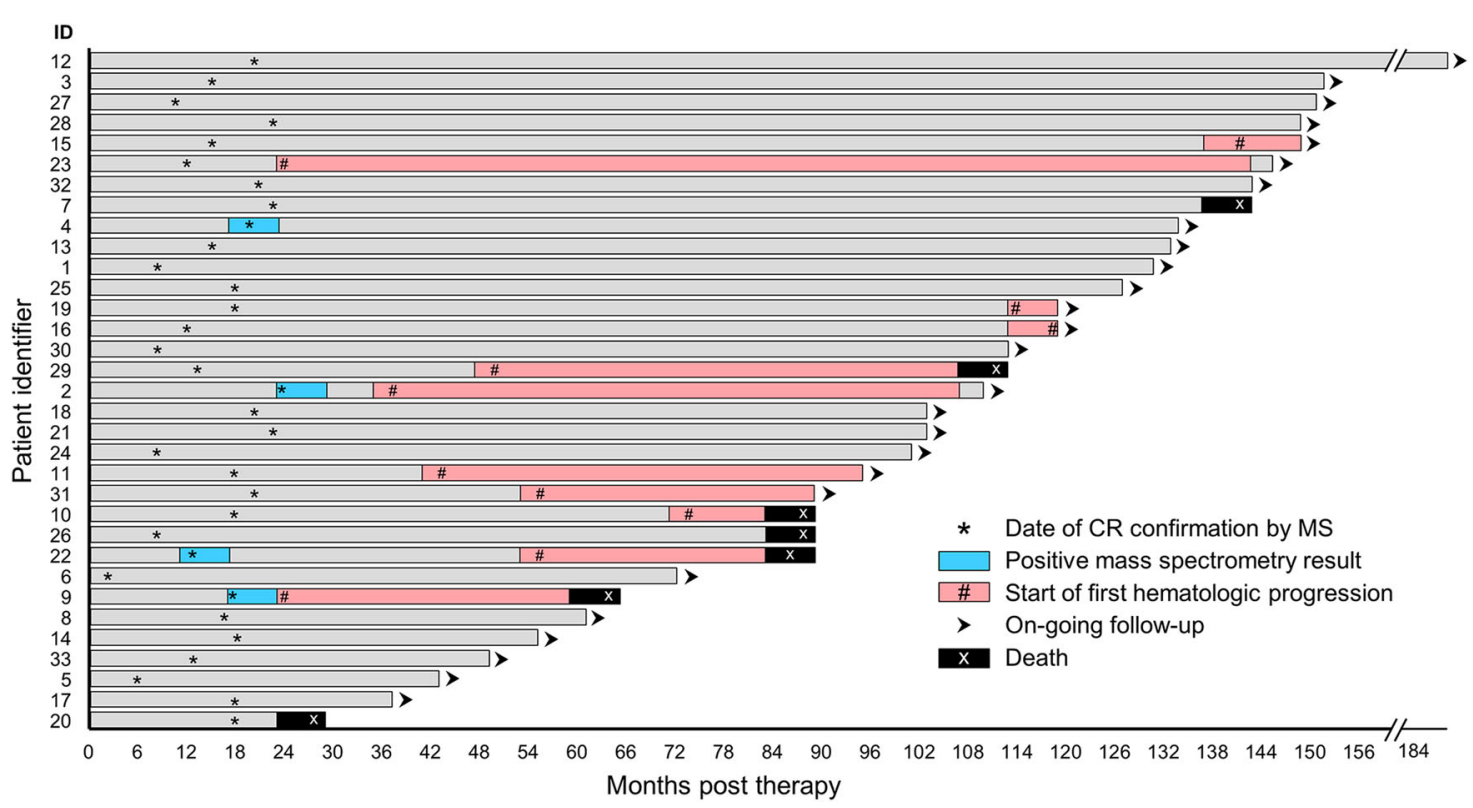

Fig. 3 Patient outcomes details.
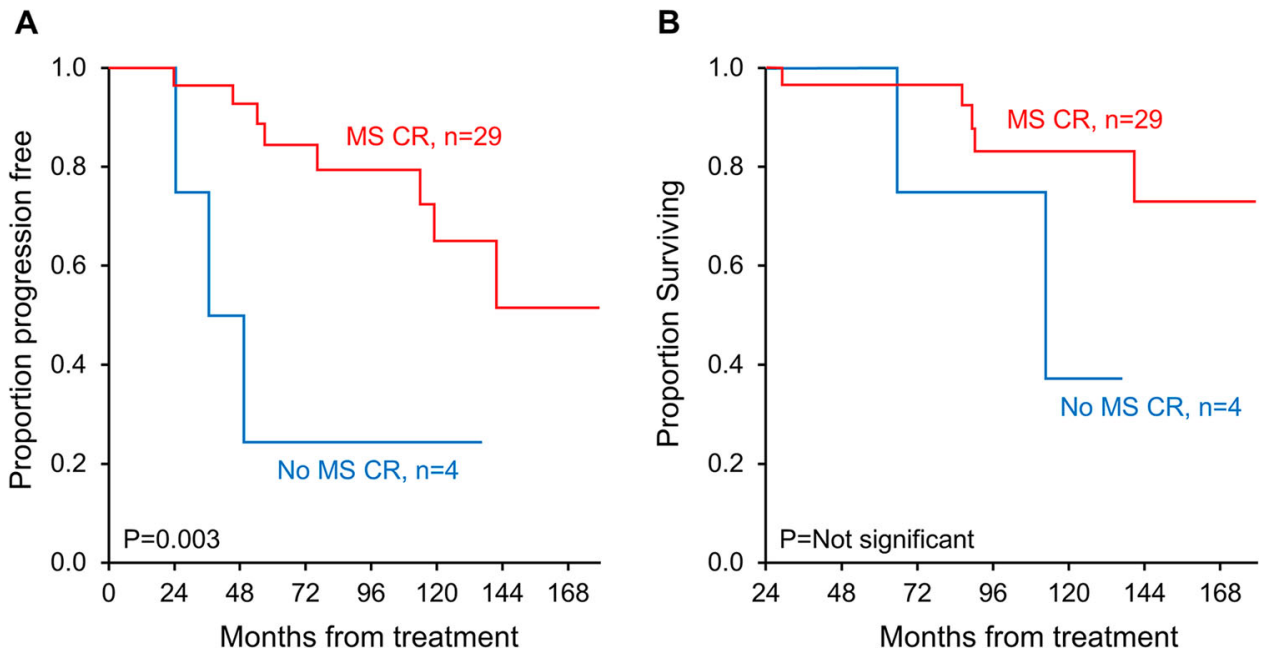

Fig. 4 Survival outcomes when using mass spectrometry. a Time to progression based on mass spectrometry analysis (either MASS-FIX or ESITOF) was positive. $\mathbf{b}$ Overall survival based on mass spectrometry analysis (either MASS-FIX or ESI-TOF) was positive.

next-generation sequencing. Current consensus response criteria exclude bone marrow as part of hematologic response $^{6,7}$, but we included bone marrow response because emerging data demonstrate that patients with a negative bone marrow by flow cytometry fare better than those without ${ }^{13-18}$. Despite the limited sample size, there was a very significant difference in progression-free survival between the MS-positive and -negative patients. Although there was a trend in better overall survival in the MS-negative patients, the study was likely underpowered to be significant.
Prior studies in AL amyloidosis and other plasma cell disorders have demonstrated the higher sensitivity and specificity of this assay ${ }^{2,5,19}$. A similar study of myeloma patients in stringent $C R$ showed greater sensitivity for these mass spectrometry techniques ${ }^{1}$. One would anticipate that the overall performance of the mass spectrometry approach would have been even better had there also been urine samples to test by mass spectrometry ${ }^{1,2}$.

Additional work in larger numbers of patients will ultimately be required to determine the range of sensitivity of mass spectroscopy of blood and urine has relative 
to the next-generation bone marrow testing, but these results are very promising.

\section{Acknowledgements}

This work was supported by JABBS Foundation, CA186781 (SPORE), Robert A. Kyle Hematologic Malignancies Fund.

\section{Author details}

'Department of Internal Medicine, Division of Hematology, Mayo Clinic Rochester, Rochester, MN, USA. ${ }^{2}$ Department of Laboratory Medicine and Pathology, Mayo Clinic Rochester, Rochester, MN, USA. ${ }^{3}$ Division of Nephrology and Hypertension, Mayo Clinic Rochester, Rochester, MN, USA

\section{Author contributions}

A.D. and D.M. designed the study, analyzed the data, wrote the first draft, and approved the final version of the manuscript; B.A. and M.K. ran the experiments revised, critically reviewed, and approved the final version of the manuscript. S.D., T.K., S.K.K., N.L., E.M., F.K.B., R.W., R.A.K., M.Q.L., D.D., P.K., W.I.G., R.S.G., S.R.H., Y. L.H., A.F., M.H., D.J., J.A.L., S.Z., S.J.R., V.S.R., and M.A.G. revised the manuscript critically and approved the final version of the manuscript.

\section{Conflict of interest}

A.D.: Research funding (Celgene, Millennium, Pfizer, Alnylam), Travel grant (Pfizer); Consultancy (Intellia, Akcea, and Janssen); B.A.: no disclosure; S.D., Ph.D., has patent rights on the assay. M.K.: None. Taxiarchis vs. Kourelis: None; S.K.K.: Consultancy (Celgene, Millennium, Onyx, Janssen, and BMS); and research funding (Celgene, Millennium, Novartis, Onyx AbbVie, Janssen, and BMS); N.L.: None; E.M.: None; F.K.B.: None; R.W.: None; R.A.K.: None; M.Q.L.: Research funding (Celgene); D.D.: Research funding (Karyopharm Therapeutics, Amgen, and Millenium Pharmaceuticals); P.K.: Research funding (Takeda, Celgene, and Amgen); W.I.G.: None; R.S.G.: None; S.R.H.: None; Y.L.H.: None; A.F.: None; M.H.: None; D.J.: None; J.A.L.: None; Yi Lin: None; S.Z.: None; S.J.R.: None; V.S.R.: None; M.A.G.: Consultancy (Milleniu) and honoraria (Celgene, Millenium, Onyx, Novartis, Smith Kline, Prothena, Ionis); D.M. has patent rights on the assay and has received research support from the Binding Site Ltd.

\section{Publisher's note}

Springer Nature remains neutral with regard to jurisdictional claims in published maps and institutional affiliations.

Supplementary Information accompanies this paper at (https://doi.org/ 10.1038/s41408-020-0291-8).

Received: 2 August 2019 Revised: 28 January 2020 Accepted: 13 February 2020

Published online: 25 February 2020

\section{References}

1. Mills, J. R., Barnidge, D. R., Dispenzieri, A. \& Murray, D. L. High sensitivity bloodbased M-protein detection in SCR patients with multiple myeloma. Blood Cancer J. 7, e590 (2017).
2. Milani, P. et al. The utility of MASS-FIX to detect and monitor monoclonal proteins in the clinic. Am. J. Hematol. 92, 772-779 (2017).

3. Barnidge, D. R., Dispenzieri, A., Merlini, G., Katzmann, J. A. \& Murray, D. L. Monitoring free light chains in serum using mass spectrometry. Clin. Chem. Lab. Med. 54, 1073-1083 (2016).

4. Kohlhagen, M. C. et al. Screening method for M-proteins in serum using nanobody enrichment coupled to MALDI-TOF mass spectrometry. Clin. Chem. 62, 1345-1352 (2016).

5. Mills, J. R. et al. Comprehensive assessment of M-proteins using nanobody enrichment coupled to MALDI-TOF mass spectrometry. Clin. Chem. 62, 1334-1344 (2016).

6. Palladini, G. et al. New criteria for response to treatment in immunoglobulin light chain amyloidosis based on free light chain measurement and cardiac biomarkers: impact on survival outcomes. J. Clin. Oncol. 30, 4541-4549 (2012).

7. Comenzo, R. L. et al. Consensus guidelines for the conduct and reporting of clinical trials in systemic light-chain (AL) amyloidosis. Leukemia 26, 2317-2325 (2012).

8. Vrana, J. A. et al. Classification of amyloidosis by laser microdissection and mass spectrometry based proteomic analysis in clinical biopsy specimens. Blood 114, 4957-4959 (2009).

9. Brambilla, F. et al. Reliable typing of systemic amyloidoses through proteomic analysis of subcutaneous adipose tissue. Blood 119, 1844-1847 (2012).

10. Katzmann, J. A. et al. Serum reference intervals and diagnostic ranges for free kappa and free lambda immunoglobulin light chains: relative sensitivity for detection of monoclonal light chains. Clin. Chem. 48, 1437-1444 (2002).

11. Morice, W. G. et al. Novel multi-parameter flow cytometry sensitively detects phenotypically distinct plasma cell subsets in plasma cell proliferative disorders. Leukemia 21, 2043-2046 (2007).

12. Barnidge, D. R. et al. Phenotyping polyclonal kappa and lambda light chain molecular mass distributions in patient serum using mass spectrometry. J. Proteome Res. 13, 5198-5205 (2014).

13. Muchtar, E. et al. The prognostic value of multiparametric flow cytometry in AL amyloidosis at diagnosis and at the end of first-line treatment. Blood 129, 82-87 (2017).

14. Lee, H. et al. Minimal residual disease (MRD) assessment by flow cytometry after ASCT for AL amyloidosis: are we there yet? Bone Marrow Transplant 52, 915-917 (2017)

15. Sidiqi, M. H. et al. Prognostic significance of stringent complete response after stem cell transplantation in immunoglobulin light chain amyloidosis. Biol. Blood Marrow Transplant 24, 2360-2364 (2018).

16. Sidana, S. et al. The importance of bone marrow examination in patients with light chain amyloidosis achieving a complete response. Leukemia 32, 1243-1246 (2018).

17. Palladini, G. et al. Persistence of minimal residual disease by multiparameter flow cytometry can hinder recovery of organ damage in patients with AL amyloidosis otherwise in complete response. Blood 128, 3261 (2016).

18. Kastritis, E. et al. Evaluation of minimal residual disease using nextgeneration flow cytometry in patients with AL amyloidosis. Blood Cancer J. 8, 46 (2018)

19. Mills, J. R. et al. A universal solution for eliminating false positives in myeloma due to therapeutic monoclonal antibody interference. Blood 132, 670-672 (2018). 